\title{
SYMMETRY OF DICHROMATIC LINKS
}

\author{
YASUYUKI MIYAZAWA
}

(Communicated by Frederick R. Cohen)

\begin{abstract}
Let $L$ be a 1-trivial dichromatic link in $S^{3}$ and $\widetilde{L}$ its covering link. A relationship between the dichromatic link polynomials, defined by Hoste and Przytycki, of $L$ and $\widetilde{L}$ is given. As an application, it is shown that the link $7_{1}^{2}$ has no symmetries with fixed point set is either of the components.
\end{abstract}

\section{INTRODUCTION}

A 1-trivial dichromatic link in $S^{3}$ is a link having at least two components, one of which is unknotted and labeled, or colored, "1", while all other components are colored " 2 ". If $L$ is a 1-trivial dichromatic link, then we may isotope $L$ until the 1-component, that is the component colored " 1 ", is the $z$-axis union the point at infinity. If we now project the link into the $x-y$ plane, we obtain a diagram of the 2 -sublink in the punctured plane $R^{2}-\{0\}$. We may obviously use such punctured diagrams to represent 1-trivial dichromatic links. Generalizing the Jones polynomial [J], Hoste and Przytycki [HP] defined a unique polynomial invariant in $Z\left[A^{ \pm 1}, h\right]$ of unoriented 1-trivial dichromatic links as follows:

$$
d_{L}(A, h)=\left(-A^{3}\right)^{-\mathrm{sw}(D)}\langle D\rangle,
$$

where $D$ is any punctured diagram of the link $L$, and $\langle D\rangle$ is the invariant of $D$ determined by the following properties:

$$
\begin{aligned}
& \langle\cdot \bigcirc\rangle=1, \\
& \langle\odot\rangle=h, \\
& \langle\times\rangle=A\langle)(\rangle+A^{-1}\langle\asymp\rangle, \\
& \langle\cdot \bigcirc K\rangle=-\left(A^{2}+A^{-2}\right)\langle\cdot K\rangle, K \neq \varnothing, \\
& \langle\odot K\rangle=-\left(A^{2}+A^{-2}\right) h\langle\cdot K\rangle, K \neq \varnothing,
\end{aligned}
$$

and $\operatorname{sw}(D)$ is the self writhe of $D$, that is, the sum of the signs of those crossings between strands belonging to the same component. Here we follow Kauffman's notation $[\mathrm{K}]$ with the additional convention of marking the puncture with a dot. If $L$ is a 1-trivial dichromatic link, we denote the 1-component by $L_{1}$ and the

Received by the editors December 5, 1989.

1980 Mathematics Subject Classification (1985 Revision). Primary 57M25.

Key words and phrases. Link, dichromatic link polynomial invariant, wrapping number. 
2-sublink by $L_{2}$. Let $N\left(L_{1}\right)$ be a tubular neighborhood of $L_{1}$. Let $\operatorname{wr}(L)$ be the wrapping number of $L_{2}$ in the solid torus $V=S^{3}-\stackrel{\circ}{N}\left(L_{1}\right)$, that is, the minimal geometric intersection number of $L_{2}$ with any meridian disk of $V$. In [HP] they show

$$
\operatorname{deg}_{h} d_{L} \leq \operatorname{wr}(L)
$$

where $\operatorname{deg}_{h} d_{L}$ is the highest degree of $h$ appearing in $d_{L}$. We consider the $p$ fold cyclic branched cover $q_{p}: S^{3} \rightarrow S^{3}$ branched over $L_{1}$. Then the upstairs branch set $\widetilde{L_{1}}$ and the preimage $\widetilde{L_{2}}=q_{p}^{-1}\left(L_{2}\right)$ again constitute a 1-trivial dichromatic link $\widetilde{L}=\widetilde{L_{1}} \cup \widetilde{L_{2}}$. Then we have the following:

Theorem 1. Let $L$ be a 1-trivial dichromatic link. Suppose $\operatorname{wr}(L)=m$. Let $f(A)$ be the coefficient of $h^{m}$ in $d_{L}$. Then the coefficient of $h^{m}$ in $d_{\widetilde{L}}$ equals $A^{6 r} \delta^{-(p-1)(m-1)}\{f(A)\}^{p}$, where $r \in Z, \delta=-\left(A^{2}+A^{-2}\right)$, and $f(A) \in Z\left[A^{ \pm 1}\right]$. In particular, if $L_{2}$ and $\widetilde{L_{2}}$ are knots, then $r=0$.

Concerning the wrapping numbers of $L$ and $\widetilde{L}$, we have:

Proposition 1. Let $L$ be a 1-trivial dichromatic link. Then the wrapping number of $\widetilde{L_{2}}$ equals the wrapping number of $L_{2}$.

Combining Theorem 1, Proposition 1, and (6), we obtain:

Theorem 2. Let $L$ be a 1-trivial dichromatic link. Then $\operatorname{deg}_{h} d_{L}=\operatorname{wr}(L)$ if and only if $\operatorname{deg}_{h} d_{\widetilde{L}}=\operatorname{wr}(\widetilde{L})$.

If $L$ is a 1-trivial dichromatic link, we say that $L$ admits a $Z_{p}$-action fixing $L_{1}$ if there exists a $Z_{p}$-action of $S^{3}$ that preserves $L$ and has fixed point set $L_{1}$.

As a corollary of Theorems 1 and 2, we have:

Corollary 1. Let $L$ be a 1-trivial dichromatic link satisfying $\operatorname{deg}_{h} d_{L}=\operatorname{wr}(L)$. Let $p$ be an integer that is more than 1 . If $L$ admits a $Z_{p}$-action fixing $L_{1}$, there exist an integer $r$ and a Laurent polynomial $g(A)$ such that the coefficient of $h^{\operatorname{wr}(L)}$ in $d_{L}$ is $\left.A^{6 r} \delta^{-(p-1)(\operatorname{wr}(L)-1)}\{g(A))\right\}^{p}$.

If a 1-trivial dichromatic link $L$ admits a $Z_{p}$-action fixing $L_{1}$, then it is shown in [HP] that

$$
d_{L}(A, h) \equiv d_{L}\left(A^{-1}, h\right) \bmod \left(A^{4 p}-1, p\right)
$$

for $L$, where $p$ is prime. If $L$ is a link that is both 1-trivial and 2-trivial, then we can also consider a $Z_{p}$-action on $S^{3}$ with fixed point set $L_{2}$. Using this, they show that the link $L=7_{6}^{2}[\mathrm{R}$, Appendix C] as shown in Figure 1 admits no $Z_{p}$-actions fixing $L_{1}$ for $p>2$ or fixing $L_{2}$ for $p>3$. Using Corollary 1 , we can show that $7_{6}^{2}$ admits no $Z_{p}$-action for any integer $p \geq 2$ such that the fixed point set is either $L_{1}$ or $L_{2}$. Other examples are also given in $\S 4$. In $\S 2$ we prove Theorem 1. In $\S 3$ we prove Proposition 1. 


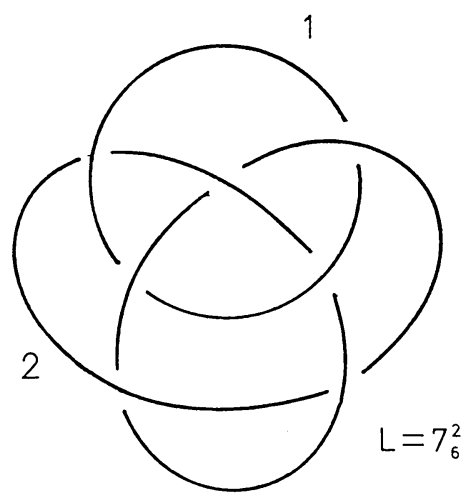

FIGURE 1

\section{Proof of TheOREM 1}

We now recall "state models" by Kauffman [K]. Let $D$ be a diagram of a link $L$. If $U$ is the underlying planar graph for $D$, then a state of $U$ is a choice of splitting marker for every vertex of $U$. Let $S$ be a state for a diagram $D$ and $D(S)$ the diagram obtained from $D$ by splitting the state $S$.

If we consider the diagrams of links, we can define the wrapping number of them as follows: Let $D$ be a punctured diagram of $L$. We define $\operatorname{wr}(D)$, the wrapping number of $D$, to be the minimal intersection number between $D$ and any ray emanating from the puncture and extending to infinity.

Lemma 1. Let $D$ be a punctured diagram of a 1-trivial dichromatic link $L$ with $\operatorname{wr}(D)=m$. Then there exists a state $S$ of $D$ satisfying $\operatorname{deg}_{h}\langle D(S)\rangle=m$.

Proof. Since $\operatorname{wr}(D)=m$ we may picture $D$ as in Figure 2. We induct on $m$. If $m=0$ or 1 , then any state $S$ of $D$ suffices. If $m>1$ then consider the

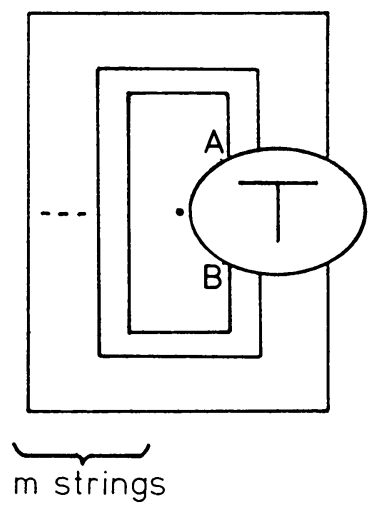

FIGURE 2 
points labelled $A$ and $B$ in Figure 2. It is possible to smooth some subset of the crossings of $D$ so that the string entering $T$ at $A$ then exits at $B$, has no crossings, and is part of an innermost circle of nontrivial ones in the resulting diagram $D^{\prime}$, where a nontrivial circle is a simple closed curve that is not nullhomotopic in $R^{2}-\{0\}$. To see this, simply enter $T$ at $A$ and smooth each crossing as it is encountered so as to always turn to the right. If this process does not force one to exit at $B$ then $\operatorname{wr}(D)<m$. Now $D^{\prime}=D_{1}^{\prime} \cup D_{2}^{\prime}$ where $D_{1}^{\prime}$ is the innermost circle mentioned above and $D_{2}^{\prime}$ has wrapping number $m-1$. By induction and property 5 of \langle\rangle$, D^{\prime}$ has a state $S^{\prime}$ such that $\operatorname{deg}_{h}\left\langle D^{\prime}\left(S^{\prime}\right)\right\rangle=m$. Now let $S$ be the extension of $S^{\prime}$ to $D$ obtained by incorporating the original smoothings used to produce $D^{\prime}$ from $D$.

Let $D$ be a diagram of $L$ as shown in Figure 2. Let $U$ be the underlying planar graph for $D$ and $U_{T}$ the subgraph of $U$ for a tangle $T$. We may regard a state $S$ for $D$ as a state for $T$, that is, a choice of splitting marker for every vertex of $U_{T}$ is $S$. Let $T(S)$ be the tangle obtained from $T$ by splitting the state $S$. Then we easily obtain:

Lemma 2. Let $L$ be a 1-trivial dichromatic link with $\operatorname{wr}(L)=m \geq 1$ and $D a$ punctured diagram of $L$ as shown in Figure 2. Let $S$ be a state for $D$. Then the following two conditions are equivalent:

(1) $\operatorname{deg}_{h}\langle D(S)\rangle=m$

(2) $T^{\prime}(S)$ is a trivial m-braid,

where $T^{\prime}(S)$ is the tangle obtained from $T(S)$ by removing all the trivial components contained entirely in $T(S)$.

Remark. Lemma 2 really is just a statement about the bracket polynomial of a diagram with no crossings. Namely the following lemma:

Lemma $2^{\prime}$. Let $D_{t, n}$ be a punctured diagram having no crossings, $t$ trivial circles, and $n$ nontrivial circles. Then $\left\langle D_{t, n}\right\rangle=\delta^{t+n-1} h^{n}$.

This lemma is essentially the same thing as Lemma 2.

We define $\langle D \mid S\rangle$ for a diagram $D$ and a state $S$ by the formula

$$
\langle D \mid S\rangle=A^{n-2 i},
$$

where $n$ is the number of crossings of $D$ and $i$ is the number of state markers corresponding to splittings which join the regions labelled $A^{-1}$ in Figure 3.

Lemma 3. Let $L$ be a 1-trivial dichromatic link. If $\operatorname{wr}(L)=m$, then the coefficient of $h^{m}$ in $d_{L}$ has the form $\delta^{m-1} f(A)$, where $f(A) \in Z\left[A^{ \pm 1}\right]$.

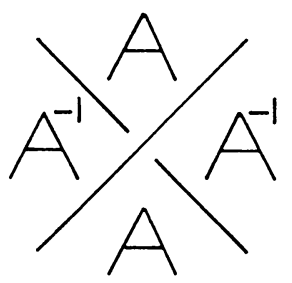

Figure 3 
Proof. Since $\operatorname{wr}(L)=m$, a diagram $D$ of $L$ can be drawn as in Figure 2. Then

$$
\langle D\rangle=\sum_{S}\langle D \mid S\rangle\langle D(S)\rangle,
$$

where this summation is over all states of the diagram. Now we compute the coefficient of $h^{m}$ in $d_{L}$. We need only consider the states

$$
\mathscr{S}=\left\{S \mid \operatorname{deg}_{h}\langle D(S)\rangle=m\right\},
$$

which by Lemma 1 is nonempty. Let $|D(S)|$ be the number of the trivial components in $D(S)$ (recall Lemma $2^{\prime}$ ). Let $D^{\prime}(S)$ be the diagram obtained from $D(S)$ by removing all the trivial components contained entirely in $T(S)$. If $m=0$, then $\langle D(S)\rangle=\delta^{|D(S)|-1}$. If $m \geq 1$, then

$$
\langle D(S)\rangle=\delta^{|D(S)|}\left\langle D^{\prime}(S)\right\rangle=\delta^{|D(S)|+(m-1)} h^{m} .
$$

So the coefficient of $h^{m}$ in $\langle D\rangle$ is given by

$$
\sum_{S \in \mathscr{S}}\langle D \mid S\rangle \delta^{|D(S)|+(m-1)}=\delta^{m-1} \sum_{S \in \mathscr{S}}\langle D \mid S\rangle \delta^{|D(S)|} .
$$

Hence the coefficient of $h^{m}$ in $d_{L}$ is

$$
\left(-A^{3}\right)^{-\operatorname{sw}(D)} \delta^{m-1} \sum_{S \in \mathscr{S}}\langle D \mid S\rangle \delta^{|D(S)|}=\delta^{m-1}\left\{\left(-A^{3}\right)^{-\operatorname{sw}(D)} \sum_{S \in \mathscr{S}}\langle D \mid S\rangle \delta^{|D(S)|}\right\} .
$$

Putting $f(A)=\left(-A^{3}\right)^{-\mathrm{sw}(D)} \sum_{S \in \mathscr{S}}\langle D \mid S\rangle \delta^{|D(S)|}$, we obtain the desired formula.

If $D$ is a diagram of $L$ as shown in Figure 2, then the diagram $\widetilde{D}$ given in Figure 4 is that of $\widetilde{L}$, where each $T_{i}, 1 \leq i \leq p$, is a copy of $T$ and $\widetilde{T}$ is the sum of $T_{1}, T_{2}, \ldots, T_{p}$. Let $U$ be the underlying planar graph for $\widetilde{D}$ and $U_{T_{i}}$ subgraph of $U$ for $T_{i}$. In Figure 4 let $S_{1}, S_{2}, \ldots, S_{p}$ be the states for the tangles $T_{1}, T_{2}, \ldots, T_{p}$, respectively. The union of $S_{1}, S_{2}, \ldots, S_{p}$ defines a state $S$ for the diagram $\widetilde{D}$, which we denote by $S=\left(S_{1}, S_{2}, \ldots, S_{p}\right)$. We may also regard $S$ as a state for $\widetilde{T}$. Let $T_{i}^{\prime}\left(S_{i}\right)$ (resp. $\widetilde{T}^{\prime}(S)$ ) be the tangle obtained from $T_{i}\left(S_{i}\right)$ (resp. $\widetilde{T}(S)$ ) by removing all the trivial components contained entirely in $T_{i}\left(S_{i}\right)$ (resp. $\widetilde{T}(S)$ ). Then we have:

Lemma 4. Let $L$ be a 1-trivial dichromatic link with $\operatorname{wr}(L)=m \geq 1$ and $\widetilde{D} a$ punctured diagram of $\widetilde{L}$ as shown in Figure 4 . Let $S$ be a state for $\widetilde{D}$. Then the following three conditions are equivalent:

(1) For each $i, 1 \leq i \leq p, T_{i}^{\prime}\left(S_{i}\right)$ is a trivial $m$-braid;

(2) $\widetilde{T}^{\prime}(S)$ is a trivial $m$-braid;

(3) $\operatorname{deg}_{h}\langle\widetilde{D}(S)\rangle=m$.

Let $L$ be a 1-trivial dichromatic link. Let $D$ be a diagram of $L$ as shown in Figure 2 and $\widetilde{D}$ the diagram of $\widetilde{L}$ as shown in Figure 4. Suppose $L_{2}$ is an oriented link having $t$ components: $L_{2}=K_{1} \cup K_{2} \cup \cdots \cup K_{t}$. Let $\widetilde{K}_{u}$ be the preimage of $K_{u}, 1 \leq u \leq t$. We also denote the components of $\widetilde{K}_{u}$, $1 \leq u \leq t$, by $K_{u, 1}, K_{u, 2}, \ldots, K_{u, n_{u}}$, so $\widetilde{L_{2}}=K_{1,1} \cup \cdots \cup K_{1, n_{1}} \cup \cdots \cup K_{t, 1} \cup$ $\cdots \cup K_{t, n_{t}}$, where $K_{u, i}$ is oriented so that it induces the same orientation on $K_{u}$ downstairs. Putting $r=\sum_{u=1}^{t} \sum_{i \neq j} l k\left(K_{u, i}, K_{u, j}\right)$, we easily obtain: 


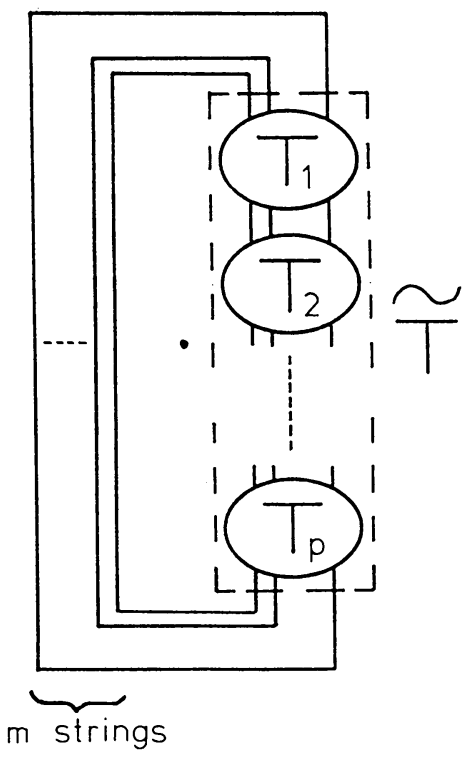

FIGURE 4

Lemma 5. $\operatorname{sw}(\widetilde{D})=\operatorname{psw}(D)-2 r$.

Proof of Theorem 1. Let $D$ be a diagram as shown in Figure 2 and $\widetilde{D}$ the diagram as shown in Figure 4. In Figure 4 let $S_{1}, S_{2}, \ldots, S_{p}$ be the states for the tangles $T_{1}, T_{2}, \ldots, T_{p}$, respectively. Let $S=\left(S_{1}, S_{2}, \ldots, S_{p}\right)$. By Lemma 1 , there exists a state $S=\left(S_{1}, S_{2}, \ldots, S_{p}\right)$ satisfying $\operatorname{deg}_{h}\langle\widetilde{D}(S)\rangle=m$. In case $m=0$ it is easy. Suppose $m \geq 1$. By Lemmas 2 and 4 , in order to compute the coefficient of $h^{m}$ in $d_{\widetilde{L}}$, we may only consider the states

$\widetilde{\mathscr{S}}=\left\{S \mid \operatorname{deg}_{h}\langle\widetilde{D}(S)\rangle=m\right\}=\left\{\left(S_{1}, S_{2}, \ldots, S_{p}\right) \mid \operatorname{deg}_{h}\left\langle D\left(S_{i}\right)\right\rangle=m, 1 \leq i \leq p\right\}$, where we regard $S_{i}, 1 \leq i \leq p$, as a state for $D$. Moreover by Lemma 4 , we have

$$
|\widetilde{D}(S)|=\sum_{i=1}^{p}\left|D\left(S_{i}\right)\right|, \quad\langle\widetilde{D} \mid S\rangle=\prod_{i=1}^{p}\left\langle D \mid S_{i}\right\rangle .
$$

The coefficient of $h^{m}$ in $\langle\widetilde{D}\rangle$ is given by

$$
\begin{aligned}
\sum_{S \in \widetilde{\mathscr{S}}}\langle\widetilde{D} \mid S\rangle \delta^{|\widetilde{D}(S)|+(m-1)} & =\delta^{m-1} \sum_{\left(S_{1}, S_{2}, \ldots, S_{p}\right) \in \tilde{\mathscr{S}}}\left(\prod_{i=1}^{p}\left\langle D \mid S_{i}\right\rangle\right) \delta^{\sum_{i=1}^{p}\left|D\left(S_{i}\right)\right|} \\
& =\delta^{m-1}\left(\sum_{S_{i} \in \mathscr{S}_{i}}\left\langle D \mid S_{i}\right\rangle \delta^{\left|D\left(S_{i}\right)\right|}\right)^{p},
\end{aligned}
$$

where $\mathscr{S}_{i}=\left\{S_{i} \mid \operatorname{deg}_{h}\left\langle D\left(S_{i}\right)\right\rangle=m\right\}$. Then by Lemma 5 the coefficient of $h^{m}$ in 
$d_{\widetilde{L}}$ is

$$
\begin{aligned}
& \left(-A^{3}\right)^{-\mathrm{sw}(\widetilde{D})} \delta^{m-1}\left(\sum_{S_{i} \in \mathscr{S}_{i}}\left\langle D \mid S_{i}\right\rangle \delta^{\left|D\left(S_{i}\right)\right|}\right)^{p} \\
& =\left(-A^{3}\right)^{-\mathrm{psw}(D)+2 r} \delta^{m-1}\left(\sum_{S_{i} \in \mathscr{S}_{i}}\left\langle D \mid S_{i}\right\rangle \delta^{\left|D\left(S_{i}\right)\right|}\right)^{p} \\
& =A^{6 r} \delta^{m-1}\left\{\left(-A^{3}\right)^{-\mathrm{sw}(D)} \sum_{S_{i} \in \mathscr{S}_{i}}\left\langle D \mid S_{i}\right\rangle \delta^{\left|D\left(S_{i}\right)\right|}\right\}^{p} .
\end{aligned}
$$

Putting $g(A)=\left(-A^{3}\right)^{-\operatorname{sw}(D)} \sum_{S_{i} \in \mathscr{S}_{i}}\left\langle D \mid S_{i}\right\rangle \delta^{\left|D\left(S_{i}\right)\right|}$, we obtain the formula by Lemma 3.

\section{Proof of Proposition 1}

In case $m=0$, it is obvious. Suppose $\operatorname{wr}(L)=m \geq 1$. We may assume that a disk $D_{0}$ giving the wrapping number of $L_{2}$ is a standard meridian disk of $V=S^{3}-\stackrel{\circ}{N}\left(L_{1}\right)$. We denote the preimage of $D_{0}$ in $\widetilde{V}=q_{p}^{-1}(V)$ by $\widetilde{D_{1}}, \widetilde{D_{2}}, \ldots, \widetilde{D_{p}}$. Suppose $\widetilde{D_{1}}$ is not a disk that gives the wrapping number of $\widetilde{L_{2}}$. Let $\widetilde{C}$ be a circle obtained by moving $\partial \widetilde{D_{1}}$ slightly on $\partial \widetilde{V}$. Hence $\widetilde{C} \cap \partial \widetilde{D_{1}}=\varnothing$. We consider properly embedded disks $\mathscr{D}=\{D \mid \partial D=\widetilde{C}\}$ in $\widetilde{V}$ satisfying:

(1) $D$ intersects $\widetilde{L_{2}}$ transversally in less than $m$ points;

(2) $D$ intersects $\widetilde{D_{1}} \cup \widetilde{D_{2}} \cup \cdots \cup \widetilde{D_{p}}$ transversally, and hence the intersection consists of disjoint circles; and

(3) $D \cap\left(\widetilde{D_{1}} \cup \widetilde{D_{2}} \cup \cdots \cup \widetilde{D_{p}}\right) \cap \widetilde{L_{2}}=\varnothing$.

Let $n(D)$ be the number of circles of (2). Let $\bar{D}$ be a disk such that $n(\bar{D}) \leq$ $n(D)$ for any $D \in \mathscr{D}$. Suppose $n(\bar{D})=0$. Then $\bar{D}$ lies in a fundamental region, which is one of the $p$ regions divided by $\widetilde{D_{1}}, \widetilde{D_{2}}, \ldots, \widetilde{D_{p}}$. Hence $q_{p}(\bar{D})$ is a disk having no singularities in $V$, and we have $\#\left\{q_{p}(\bar{D}) \cap L_{2}\right\}=$ $\#\left\{\bar{D} \cap \widetilde{L_{2}}\right\}<m=\#\left\{D_{0} \cap L_{2}\right\}$. This is a contradiction. Suppose $n(\bar{D}) \neq 0$. Let $C_{0}$ be one of innermost circles in $\bar{D}$ and $C_{0} \subset \widetilde{D_{k}}$, and let $D^{\prime}$ be the disk in $\bar{D}$ with $\partial D^{\prime}=C_{0}$. Hence $D^{\prime}$ is a disk in $\widetilde{V}$ and is embedded in a fundamental region $W$. Let $\widehat{D_{k}}$ be the disk in $\widetilde{D_{k}}$ with $\partial \widehat{D_{k}}=C_{0}$. See Figure 5. If $\#\left\{D^{\prime} \cap \widetilde{L_{2}}\right\}<\#\left\{\widehat{D_{k}} \cap \widetilde{L_{2}}\right\}$, then for the disk $\widetilde{D}=\left(\widetilde{D_{k}}-\widehat{D_{k}}\right) \cup D^{\prime}$, we have the following inequality $\#\left\{\widetilde{D} \cap \widetilde{L_{2}}\right\}<\#\left\{\widetilde{D_{k}} \cap \widetilde{L_{2}}\right\}$. Hence \#\{qp $\left.(\widetilde{D}) \cap L_{2}\right\}$

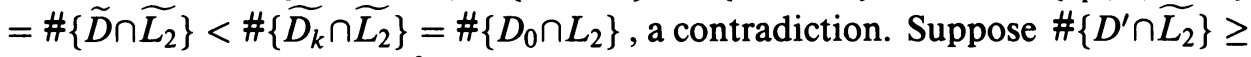
$\#\left\{\widehat{D_{k}} \cap \widetilde{L_{2}}\right\}$. Then $\left(\bar{D}-\stackrel{\circ}{D}^{\prime}\right) \cup \widehat{D_{k}}$ is an immersed disk in $\widetilde{V}$. We denote it by $\widetilde{D}$. Note that $\#\left\{\widetilde{D} \cap \widetilde{L_{2}}\right\}<m$. We consider the cases:

Case 1. $\widetilde{D}$ is a disk having no singularities.

Case 2. $\widetilde{D}$ has singularities.

In Case 1 we can push $\widetilde{D}$ slightly away from $\widetilde{D_{k}}$ near $\widehat{D_{k}}$. This shows that $n(\widetilde{D})<n(\bar{D})$, which is a contradiction. 


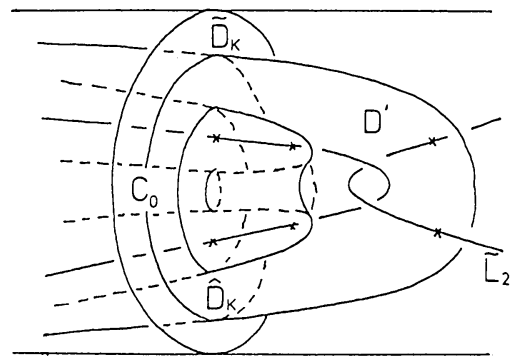

FIGURE 5

In Case 2 we note that singularities are circles $C_{1}, C_{2}, \ldots, C_{s}$ in $\widehat{D_{k}}$ and consist of double points. Let $N\left(C_{i}\right)$ be a tubular neighborhood of $C_{i}, 0 \leq i \leq$ $s$, in $\widetilde{V}$. We consider $N\left(C_{0}\right), N\left(C_{1}\right), \ldots, N\left(C_{s}\right)$ such that $N\left(C_{i}\right) \cap \widetilde{L_{2}}=\varnothing$, $0 \leq i \leq s$, and $N\left(C_{i}\right) \cap N\left(C_{j}\right)=\varnothing, i \neq j$. By a suitable cut and paste operation in $N\left(C_{1}\right)$ as Hempel's Lemma (see [H, Lemma 4.6]), we can obtain a new disk $\widetilde{D}^{\prime}$ whose singularities are $C_{2}, \ldots, C_{s}$. Then the number of intersection of $\widetilde{D}^{\prime}$ and $\widetilde{L_{2}}$ equals that of $\widetilde{D}$ and $\widetilde{L_{2}}$ since $N\left(C_{1}\right) \cap \widetilde{L_{2}}=\varnothing$. Using this repeatedly, we have a nonsingular disk $\widehat{D}$ with $\#\left\{\widehat{D} \cap \widetilde{L_{2}}\right\}=\#\left\{\widetilde{D} \cap \widetilde{L_{2}}\right\}<m$. We can push the part $\widehat{D} \cap \widehat{D_{k}}$ of $\widehat{D}$ slightly away from $\widetilde{D_{k}}$. This shows that $n(\widehat{D})<n(\bar{D})$, which is a contradiction. It follows that $\widetilde{D_{1}}$ is a disk giving the wrapping number of $\widetilde{L_{2}}$.

\section{Applications}

Using Corollary 1 , we prove that the link $7_{1}^{2}[\mathrm{R}$, Appendix $\mathrm{C}]$ as shown in Figure 6 admits no $Z_{p}$-action for any integer $p \geq 2$ such that the fixed point set is each of the trivial components, and that the link $8_{9}^{3}[R$, Appendix C] as shown in Figure 7 admits no $Z_{p}$-action for any integer $p \geq 3$ such that the fixed point set is the component colored " 1 ."

Since $L=7_{1}^{2}$ is a link that is both 1-trivial and 2-trivial, we may compute $d_{L}$ relative to either component. Call these two invariants $d_{L}^{1}$ and $d_{L}^{2}$, respectively. But $7_{1}^{2}$ is interchangeable, so $d_{L}^{1}=d_{L}^{2}$. The highest degree of $h$ in $d_{L}^{1}$ is 3 . Thus

$$
\operatorname{deg}_{h} d_{L}^{1}=\operatorname{wr}\left(L^{(2)}\right)=3,
$$

where $\operatorname{wr}\left(L^{(2)}\right)$ is the wrapping number of $L_{2}$ in the solid torus $S^{3}-\stackrel{\circ}{N}\left(L_{1}\right)$. Suppose there is a $Z_{p}$-action with fixed point set $L_{1}$ for an integer $p \geq 2$. Then by Corollary 1 there exists a Laurent polynomial $f(A)$ such that the coefficient of $h^{3}$ in $d_{L}^{1}$ is equal to $\delta^{-2(p-1)}\{f(A)\}^{p}$. On the other hand the coefficient of $h^{3}$ in $d_{L}^{1}$ is $-A^{4}\left(A^{2}+A^{-2}\right)^{2}\left(A^{4}-1\right)$. Thus

$$
\left\{f(A) / \delta^{2}\right\}^{p}=-A^{4}\left(A^{4}-1\right) .
$$

The right-hand term has the factor $A-1$. Hence the left-hand term must have the factor $A-1$, so it has the factor $(A-1)^{p}$. But the right-hand term does not have this factor. This is a contradiction. Next we consider $L=8_{9}^{3}$. The highest degree of $h$ in $d_{L}^{1}$ is 2 . Thus

$$
\operatorname{deg}_{h} d_{L}^{1}=\operatorname{wr}\left(L^{(2)}\right)=2 .
$$




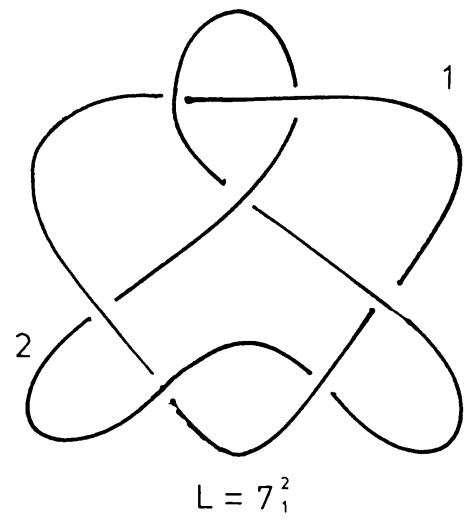

FIGURE 6

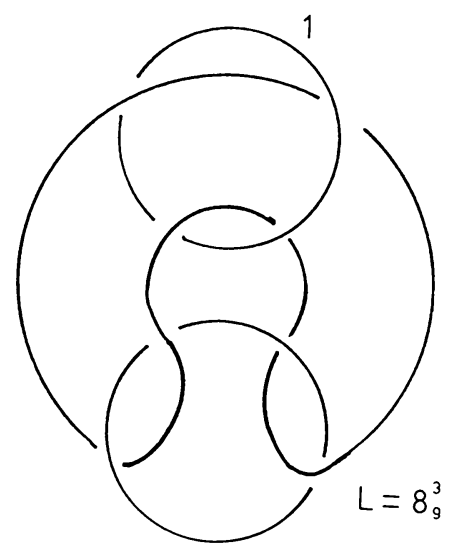

Figure 7

Suppose there is a $Z_{p}$-action with fixed point set $L_{1}$ for an integer $p \geq 2$. Since the coefficient of $h^{2}$ in $d_{L}^{1}$ is $-\left(A^{2}+A^{-2}\right)\left(A^{4}-1\right)^{2}$, by Corollary 1

$$
A^{6 r} \delta^{-(p-1)}\{f(A)\}^{p}=-\left(A^{2}+A^{-2}\right)\left(A^{4}-1\right)^{2},
$$

for some $f(A) \in Z\left[A^{ \pm 1}\right]$ and for some integer $r$. Thus

$$
\{f(A) / \delta\}^{p}=A^{-6 r}\left(A^{4}-1\right)^{2} .
$$

This is impossible for $p \geq 3$. If $p=2$, then $8_{9}^{3}$ admits a $Z_{2}$-action with quotient the Whitehead's link.

Remark. $7_{6}^{2}=L=L_{1} \cup L_{2}$ admits no $Z_{p}$-action for any integer $p \geq 2$ such that the fixed point set is either $L_{1}$ or $L_{2}$. Suppose $7_{6}^{2}$ admits a $Z_{p}$-action. Then there exists a factor link $\widehat{L}=\widehat{L_{1}} \cup \widehat{L_{2}}$ of $L$. Since $L_{2}$ is a knot and the linking number $1 \mathrm{k}\left(L_{1}, L_{2}\right)$ of $L_{1}$ and $L_{2}$ is zero, $\widehat{L_{2}}$ becomes a knot and the linking number $\operatorname{lk}\left(\widehat{L_{1}}, \widehat{L_{2}}\right)$ is equal to zero. This is a contradiction by the following fact: Suppose $L=K_{1} \cup K_{2}$ is a two component link such that $K_{1}$ is 
a trivial knot and $1 \mathrm{k}\left(K_{1}, K_{2}\right)=0$. If we consider the $n$ fold cyclic branched cover of $S^{3}$ branched over $K_{1}$, then for a covering link $\widetilde{L}=\widetilde{K_{1}} \cup \widetilde{K_{2}}$ of $L$, $\widetilde{K_{2}}$ is an $n$-component link.

\section{ACKNOWLEDGMENT}

The author wishes to thank Professors Mitsuyoshi Kato and Taizo Kanenobu for their encouragement and advice and he is grateful to Masako Kobayashi who suggested that he should use Hempel's Lemma to refine his proof of Proposition 1. The author would also like to thank the referee for giving the simple proof of Lemma 1.

\section{REFERENCES}

[H] J. Hempel, 3-manifolds, Ann. of Math. Stud., no. 86, Princeton Univ. Press, Princeton, NJ, 1976.

[HP] J. Hoste and J. H. Przytycki, An invariant of dichromatic links, Proc. Amer. Math. Soc. 105 (1989), 1003-1007.

[J] V. F. R. Jones, Hecke algebra representations of braid groups and link polynomials, Ann. of Math. (2) 126 (1987), 335-388.

[K] L. Kauffman, State models and the Jones polynomials, Topology 26 (1987), 395-407.

[R] D. Rolfsen, Knots and links, Lecture Series no. 7, Publish or Perish, Berkeley, 1976.

Department of Mathematics, Kyushu University, Fukuoka, 812 Japan

Current address: Department of Mathematics, Osaka City University, Osaka, 558 Japan 